\title{
Extractive substances of fruit body golden chanterelle (cantharellus cibarius fr.) And hedgehog mushroom (hydnum repandum fr.)
}

\author{
Lidiia V. Panchak ${ }^{a}$, Lidiia Ya Antonyuk ${ }^{\mathrm{b}}$, Alina R. Zyn'c, Volodymyr Antonyuka,d* \\ ${ }^{a}$ Department of inorganic and bioinorganic chemistry of Danylo Halytsky Lviv National Medical University; Pekarska str., 69, Lviv, Ukraine, \\ ${ }^{b}$ Lviv Medical College of Postgraduate Education, Mykolaichuka str., 9, Lviv, Ukraine, 'Lviv Research Forensic Centre of MIA of Ukraine, \\ Koniushynna str., 24, Lviv, Ukraine, 'Institute of Cell Biology, National Academy of Sciences of Ukraine, 14/16 Dragomanova str., Lviv, Ukraine
}

\section{A B S T R A C T}

\begin{abstract}
The purpose of this work was to perform a comparative study of the chemical composition of Cantharellus cibarius Fr. and Hydnum repandum $F r$. and the revision of the data in the occurrence of carotenoids, ergocaliferol, tocopherols and phenolic compounds. Substances extracted with $80 \%$ ethanol from dried fruit bodies of both fungi were investigated. The fatty acids quantitative and qualitative contents were examined by Gas chromatography Mass Spectrometry (GC-MS). Lipophilic substances of hexane extract were investigated by chemical reactions and Thin-layer chromatography (TLC) after the separation of compounds from this fraction on a silica gel column. It was determined that the hexane fraction of both mushrooms contained a large amount of higher fatty acids and their esters. Linoleic acid (31.42\%) and oleic acid $(57.89 \%)$ were the dominant components in Cantharellus cibarius and Hydnum repandum accordingly. Tocopherols, carotenoids and ergocalciferol were also found in the hexane extracted fraction. The estimated content of tocopherols was $\approx 0.82 \%$ of the total mass of ethanolic extract for chanterelle and $\approx 0.22 \%$ for hedgehog mushroom, respectively. Chloroform and ethyl acetate fractions did not contain tocopherols, carotenoids and ergocalciferol and their mass was insignificant. Carbohydrates content was $\approx 54 \%$ and $\approx 49 \%$ of the total mass of ethanolic extract and the content of aminoacids was found as $\approx 4.5 \%$ and $\approx 4.7 \%$ for Cantharellus cibarius and Hydnum repandum accordingly. Flavonoids, phenolic substances and hydroxycinnamic acids were not found in the fractions of ethanol extracts of both mushrooms.
\end{abstract}

Keywords: Cantharellus cibarius Fr.; Hydnum repandum Fr.; Lipophilic substances; Carotenoids; Tocopherols

\section{INTRODUCTION}

Mushrooms are consumed as a food high in nutritive value. They contain useful for the humans vitamins, amino acids, carbohydrates and minerals. They also often contain substances that have antibiotic properties. There is literature that the ethanolic extract of Cantharellus cibarius has anthelmintic activity, in particular, effective against Fasciola infection (Nwofor et al., 2019). Investigating the effect of golden chanterelle extract on the trematodes of the Opistorchiidae family in vivo, a significant decrease in the number of helminths in the bile ducts of the liver of mice, evaluated 6 weeks after infection was demonstrated (Tsyganov et al., 2018). The intraperitoneal injection of the chanterelle methanol extract extended the life of the experimental mice with hemic hypoxia, caused by intraperitoneal administration of sodium nitrite, in
1.5 times (Khalili et al., 2014). In folk medicine chanterelles are used in treatment of stomach, liver, lungs diseases and vision improvement. They are also used in boils and abscesses and as an anthelmintic agent (Stryamets et al., 2015; Long Chen et al., 2017).

Less is known about biologically active substances of hedgehog mushroom. From the methanol extract of the fungal bodies by separating it with ethyl acetate and subsequent separation on a silica gel column cytotoxic diepxide was purified. This pale yellow waxy substance was named repandiol (Akira et al., 1992) and was especially active against human adenocarcinoma cells.

The caps of chanterelle and hedgehog mushrooms are similar in both color and shape. The fruiting bodies of both young mushrooms are very similar in color and shape

\footnotetext{
*Corresponding author:

Volodymyr Antonyuk, Department of inorganic and bioinorganic chemistry of Danylo Halytsky Lviv National Medical University; Pekarska str., 69, Lviv, Ukraine, Institute of Cell Biology, National Academy of Sciences of Ukraine, 14/16 Dragomanova str., Lviv, Ukraine.

E-mail: antonyukvo@gmail.com 
and young hedgehog mushrooms are sometimes gavered instead of chanterelles. However, both mushrooms are edible and such a mistake has no fatal consequences. In plants, the yellow color of flowers is most often due to flavonoids or carotenoids. In the literature there is information about the occuerence of flavonoids in methanolic and ethanol extracts of chanterelles (Vamanu and Nita 2014; Kozarski et al., 2015), as well as in the hexane fraction (Ebrahimzadeh et al., 2015). However, in our opinion, the conclusions given in these studies, made by the authors, are insufficiently confirmed by experiments. Flavonoids do not usually occur in mushrooms. The yellow color of their fruiting bodies may be due to other substances. For example, two yellow substances (letiporic acid and 4-dehydro-3-deoxylethyliporic acid) with structure similar to carotene were obtained from the fruiting bodies of sulphur polypore (Laetiporus sulpbureus), which is very similar to chanterelles in colour. (Davolia et al., 2005).

Contradictory data in the literature are also given regarding hydroxycinnamic acids. Using HPLC-DAD and HPLC-UV, it was concluded that there are 5 hydroxycinnamic acids (3-, 4-, and 5-O-caffeic acid, caffeic, p-coumaric acids) in chanterelles (Valentao et al., 2005). At the same time, a group of other authors, from chanterelles, also collected in Portugal, using HPLC-DAD-ESI / MS methods, flavonoids and hydroxycinnamic acid, were not found (Barros et al., 2009).

The purpose of this work was to perform a comparative study of the chemical composition of Cantharellus cibarius Fr. extractives. and Hydnum repandum Fr. and revision of the literature on the presence of carotenoids, ergocaliferol and tocopherols and phenolic compounds.

\section{MATERIALS AND METHODS}

\section{Raw material and obtaining extracts}

Fruit bodies of mushrooms were harvested in the Skole district of Lviv region. After initial purification, they were oven-dried at $55^{\circ} \mathrm{C} \pm 1{ }^{\circ} \mathrm{C}$. The powdered fruiting bodies were extracted with $80 \%$ ethanol in a hot water bath $\left(60^{\circ} \mathrm{C}\right)$ with continuous stirring for one hour in the ratio of raw material to extractant 1:10. The resulting extract was filtered, the solvent was distilled off, and the ethanol residues in the extract were removed by evaporation in a drying oven.

The resulting ethanol extracts of chanterelle and hedgehog mushroom were fractionated with organic solvents and water. Then they were sequentially extracted with hexane $\rightarrow$ chloroform $\rightarrow$ ethyl acetate $\rightarrow$ methanol $\rightarrow$ and distilled water in a ratio of 1: 10 . The solvent was distilled off and the extracts were oven-dried.
The fatty acids from the hexane extract were extracted with $80 \%$ ethanol heated to $50^{\circ} \mathrm{C}$, which was added 5 times in portions with 10 minutes stirring. The quantitative and qualitative content of higher fatty acids in this extract of chanterelle and hedgehog mushroom was investigated by GC-MS.

\section{The identification of fatty acids and related substances by GC-MS}

The identification of fatty acids and related substances in these extracts was performed according to the recommendations described earlier (Botineştean et al., 2012). We using a 6C / MS Agilent Technologies $6890 \mathrm{~N}$ / 5975 B mass spectrometer (USA) attached to the chromatographic column HP-5MS model, $30 \mathrm{~m}$ length, $0.25 \mathrm{~mm}$ diameter, filler: $95 \%$ dimethylpolysiloxane + $5 \%$ diphenylpolysiloxane; carrier gas - helium with a constant flow of $1.5 \mathrm{ml} / \mathrm{min}$. The column was washed with methanol. Gas chromatography was programmed for a temperature rise rate $15^{\circ} \mathrm{C} / \mathrm{min}$ from 75 to $300{ }^{\circ} \mathrm{C}$. The initial temperature was maintained for $1 \mathrm{~min}$ and the final temperature for $8 \mathrm{~min}$. A mass-selective detector with interface temperature $\mathrm{T}=250{ }^{\circ} \mathrm{C}$ was used. The ionization was performed by electron impact, the ionization energy was $70 \mathrm{eV}$, the ion source temperature was $230^{\circ} \mathrm{C}$; quadrupole temperature $-150^{\circ} \mathrm{C}$.

Separation of the substances on a column of silica gel Subsequent separation of the substances of hexane extract of chanterelle and hedgehog mushroom was carried out on a column of silica gel L 40/160 which was sequentially washed with a mixture of 1) chloroform: acetic acid 100: 1;2) chloroform: acetic acid: methanol - 100: 1: 10; 3) chloroform: acetic acid: methanol - 100: 1: 4; 4 chloroform: acetic acid: methanol - 100: 1: 100. Fractions (1.5 ml) were collected into accurately weighed Eppendorf tubes. The mass of the obtained fractions was determined after evaporation of the solvent.

\section{Detection of fatty acids, carotenoids, ergocalciferols and tocopherols by TLC}

The detection of fatty acids, carotenoids, ergocalciferols and tocopherols in the extracts and in the obtained fractions was carried out by thin layer chromatography (TLC) on silufol plates (Czech Republic). The chromatograms were observed in UV light and developed with detection reagents. Tocopherols on TLC were detected by spraying $56 \% \mathrm{HNO}_{3}$ and $5 \%$ phosphorus-molybdenum acid solution in alcohol, and ergocalciferols with aniline reagent (aniline: 36\% HCI - 15: 1), 70\% $\mathrm{HClO}_{4}$, and 5\% solution of $\mathrm{SbCl}_{3}$ in chloroform or isopropanol.

To detect carotenoids, chromatogram was sprayed with concentrated $\mathrm{H}_{2} \mathrm{SO}_{4}$, which developed carotenoids as blue 
spots that began to darken quickly. TLC was also treated with a solution of $\mathrm{SbCl}_{3}$ in $\mathrm{CHCl}_{3}$, followed by heating to $90^{\circ} \mathrm{C}$.

\section{Detection of flavonoids, carbohydrates, aminoacids and organic acids}

Substances of flavonoid nature were detected by chemical reactions in the initial ethanol extract and in methanol and aqueous extracts after fractionation of the extracts. In order to detect the abovementioned substances a cyanidine reaction, a reaction with an alcohol solution of $\mathrm{FeCl}_{3}, \mathrm{AlCl}_{3}$ and $\mathrm{SbCl}_{3}$, a solution of basic lead acetate and barium hydroxide were performed. For comparison, the same reactions were performed with a $1 \%$ alcohol solution of rutin.

The quantitative content of carbohydrates in methanolic and aqueous fractions was determined by reaction with a solution of L-tryptophan and concentrated $\mathrm{H}_{2} \mathrm{SO}_{4}$ in the presence of boric acid (Methods...1967).

The quantitative content of aminoacids was determined by reaction with ninghydrin (Pochinok, 1976).

The total content of organic acids in the aqueous fractions was determined by compleximetric method after precipitation them with lead salts (Pochinok, 1976).

All reagents and standards used were purchased from Sigma-Aldrich (Sigma-Aldrich GMBH, Munich, Germany). All reagents used were of analytical quality.

\section{Statistical analysis}

Each experiment was performed in triplicate. The average values were recorded. Thin layer chromatography was also performed at least three times. The data were evaluated statistically using Student's t-test, and a value of $\mathrm{p} \leq 0.05$ was considered to be statistically reliable.

\section{RESULTS AND DISCUSSIONS}

The extraction of $80 \%$ ethanol-dried fruiting bodies of chanterelles and hedgehog mushroom in both cases produced an oily mass of carrot yellow (chanterelles) and slightly darker light brown (hedgehog mushroom ). The yield of extractives was $13.59 \%$ in chanterelles and $28.62 \%$ in hedgehog mushroom (by weight of dried fruit bodies, the average value) (Table 1).

Thus, substances extracted with $80 \%$ ethanol from yellow hedgehog are 2 times more than chanterelles.

Extracted with 80\% ethanol substances were separated into fractions by extraction with organic solvents and water. The results of these experiments are presented in Table 2.

In both fungi, the water-soluble substances of ethanol extracts were about $50 \%$ by weight. At the same time, substances re-extracted with hexane in these extracts were $31.2 \%$ for chanterelles and $20.5 \%$ for hedgehog mushrooms. The latter are also well soluble in chloroform.

The next stage of the investigation was to determine from which components consist of the fractions. Carbohydrates, organic acids, amino acids, and phenolic compounds, including flavonoids, can be extracted by water and methanol. Therefore, we conducted further research to identify them. However, fractions No 3, No 4 and No 5 of both mushrooms did not give a positive reaction with a solution of iron-III-chloride (phenolic compounds) and cyanidin reaction and a reaction with boron-lemon reagent (flavonoids). Fraction No. 5 with solutions of barium hydroxide and lead acetate gave a precipitate of a white (rather than yellow) color, which is characteristic of organic acids but not flavonoids. The fractions 4 and 5 do not have characteristic for flavonoids maxima in the UV spectrum at 320 - $400 \mathrm{~nm}$. At the same time, the reaction to the carbohydrates with anthron and sulfuric acid was positive. Determination of the quantitative content of hexoses by reaction with a solution of L-tryptophan and concentrated $\mathrm{H}_{2} \mathrm{SO}_{4}$ in the presence of boric acid (Methods of carbohydrate chemistry, 1967) showed that in these fractions they are the main components. There is also high content of amino acids in these fractions (Table 3).

Preliminary identification of substances of fractions No. 1-3 was carried out by TLC on silufol plates. The preferred system for separation was the following: chloroform: acetic acid: methanol - 100: 1: 4, and hexane: ethyl acetate: methanol - 40: 6: 1. Then they were viewed in UV light. There was found a several spots with yellow-green and blue fluorescence. But the mass of fractions No. 2-3 was insignificant, so the main focus was on the analysis of fractions No. 1, which were obtained from both mushrooms.

However, according to some literature sources (Valentao et al., 2005; Barros et al., 2008; Vamanu and Nita

$\begin{aligned} & \text { Table 1: Yield of substances extracted with } \mathbf{8 0} \% \text { ethanol } \\
& \text { from the dried fruiting bodies of chanterelles and hedgehog } \\
& \text { mushrooms }\end{aligned}$
\begin{tabular}{lccc}
\hline Experiment number & $\%$ of the extracted substances \\
\cline { 2 - 3 } & Chanterelles & Hedgehog mushrooms \\
\hline 1 & 13.78 & 28.68 \\
2 & 12.96 & 27.88 \\
3 & 14.02 & 29.32 \\
The average value & 13.59 & 28.62 \\
\hline
\end{tabular}

Emir. J. Food Agric • Vol $32 \bullet$ Issue $11 \bullet 2020$ 
Panchak, et al.

Table 2: The results of the fractionation of ethanol extracts of chanterelles and hedgehog mushrooms

\begin{tabular}{|c|c|c|c|c|c|c|c|c|}
\hline \multirow{3}{*}{$\begin{array}{l}\text { Mass of ethanol } \\
\text { extract, taken for } \\
\text { fractionation }\end{array}$} & \multicolumn{8}{|c|}{ Mass of extracted substances and their $\%$} \\
\hline & \multicolumn{2}{|c|}{ Hexane No 1} & \multirow{2}{*}{$\begin{array}{l}\text { Chloroform } \\
\text { No } 2\end{array}$} & \multirow{2}{*}{$\begin{array}{l}\text { Ethyl acetate } \\
\text { No } 3\end{array}$} & \multirow{2}{*}{$\begin{array}{l}\text { Methanol } \\
\text { No } 4\end{array}$} & \multirow{2}{*}{$\begin{array}{l}\text { Water } \\
\text { No } 5\end{array}$} & \multirow[t]{2}{*}{ Remainder } & \multirow[t]{2}{*}{ Total } \\
\hline & Total & No 1a & & & & & & \\
\hline $\begin{array}{l}\text { Chanterelles } \\
\text { (2034 mg) }\end{array}$ & $\begin{array}{c}634 \\
31.2 \%\end{array}$ & $\begin{array}{c}486 \\
23.9 \%\end{array}$ & $\begin{array}{c}61 \\
(3.0 \%)\end{array}$ & $\begin{array}{c}18 \\
(0.88 \%)\end{array}$ & $\begin{array}{c}195 \\
(9.6 \%)\end{array}$ & $\begin{array}{c}1023 \\
(50.3 \%)\end{array}$ & $\begin{array}{c}94 \\
(4.62 \%)\end{array}$ & $\begin{array}{c}2025 \\
(99.6 \%)\end{array}$ \\
\hline $\begin{array}{l}\text { Hedgehog mushrooms } \\
(10720 \mathrm{mg})\end{array}$ & $\begin{array}{c}2196 \\
20.5 \%\end{array}$ & $\begin{array}{c}1144 \\
10.7 \%\end{array}$ & $\begin{array}{c}564 \\
(5.3 \%)\end{array}$ & $\begin{array}{c}60 \\
(0.56 \%)\end{array}$ & $\begin{array}{c}2017 \\
(18.8 \%)\end{array}$ & $\begin{array}{l}5467 \\
(51 . \%)\end{array}$ & $\begin{array}{c}190 \\
(1.77 \%)\end{array}$ & $\begin{array}{l}10.494 \\
(98.7 \%)\end{array}$ \\
\hline
\end{tabular}

No $1 \mathrm{a}$ is the mass of substances remaining in hexane extract after re-extraction with $80 \%$ ethanol

Table 3: Carbohydrate, Organic acids and Amino acids content in fractions No 4 and No 5 of chanterelles and hedgehog mushrooms

\begin{tabular}{|c|c|c|c|c|c|}
\hline & \multirow[b]{2}{*}{ Fractions } & \multicolumn{4}{|c|}{ Content in fractions (in \%) } \\
\hline & & Carbohydrate & Amino acids & Organic acids & Total \\
\hline \multirow[t]{3}{*}{1} & Chanterelles & & & & \\
\hline & No 4 & $89 \%$ & $8.0 \%$ & $2.3 \%$ & $99.3 \%$ \\
\hline & No 5 & $90 \%$ & $7.4 \%$ & - & $99.7 \%$ \\
\hline \multirow[t]{3}{*}{2} & Hedgehog mushrooms & & & & \\
\hline & No 4 & $84 \%$ & $12 \%$ & $3.5 \%$ & $99.5 \%$ \\
\hline & No 5 & $65 \%$ & $4.8 \%$ & - & $69.8 \%$ \\
\hline
\end{tabular}

2014), chanterelles do contain hydroxycinnamic acids. It is known that they can be dissolved in ethyl acetate (fraction No 3) of methanol (fraction No 4) and water (fraction No 5), so for these components we used reacted with $\mathrm{FeCl}_{3}$ to detect them. However, the results were negative. Hydroxycinnamic acids were not detected by characteristic blue or blue fluorescence in UV light on paper chromatograms. No characteristic changes were observed in the processing of $\mathrm{NaOH}$ and $\mathrm{AlCl}_{3}$ chromatograms. This led to the conclusion that there were no hydroxycinnamic acids in the ethyl acetate, methanolic and aqueous fractions of Cantharellus cibarius and Hydnum repandum.

When spraying TLC hexane fractions (No. 1) from both mushrooms with a solution of methyl red, the red zones appear, indicating the presence of acidic compounds. The processing of TLC with a solution of copper acetate followed by repair of $\mathrm{K}_{3}\left[\mathrm{Fe}(\mathrm{CN})_{6}\right]$ gave brown spots of copper salts of fatty acids. This indicates their high content in the hexane fraction of the fruiting bodies of both fungi. For fatty acids extraction, hexane fractions of Cantharellus cibarius and Hydnum repandum were treated twice with two volumes of $80 \%$ aqueous ethanol at $50^{\circ} \mathrm{C}$. The combined ethanol extracts were evaporated and analyzed parallel with insoluble in ethanol remainder by TLC on the presence of organic acids (Fig. 1).

As can be seen with the chromatograms, extraction with $80 \%$ aqueous ethanol eliminates most of the higher fatty acids, but some quantity remain. Spraying TLC with methyl red also shows that in the hexane residue after ethanol extraction there are still substances of acid nature, but which do not respond to the formation of copper salts.
The combined extracts obtained by re-extraction with $80 \%$ ethanol from hexane fraction were evaporated and analyzed by GC-MS, and the insoluble residue was separated on a silica gel column and analyzed for detection tocopherols, carotenoids and ergocalciferol.

Analysis of aliphatic acids and their esters in hexane extracts of Cantharellus cibarius and Hydnum repandum, conducted by GC-MS gave the results presented in Table 4.

Thus, both mushrooms have a high content of unsaturated fatty acids. The dominant component in extract Cantharellus cibarius present is linolenic acid (31.42\%) and in Hydnum repandum - oleic acid (57.89\%).

Spraying chromatograms with a solution of stybium chloride in chloroform followed by heating for 10 minutes at $70^{\circ} \mathrm{C}$ showed blue zones, which can give carotenoids and ergocalciferols. Similar areas in the same places appear when spraying TLC with concentrated sulfuric acid, which is also typical of carotenoids.

Also in these fractions, when sprayed with 56\% nitric acid, followed by holding at $90^{\circ} \mathrm{C}$ for $30 \mathrm{~min}$ appear areas that are colored in pink-brown are characteristic of tocopherols. However, these reactions are not specific enough and the composition of the fractions is quite complex. Therefore, we have separated the substances of the fractions on a column of silica gel under the conditions described in the "materials and methods". The separation chart is presented in Fig. 2.

As you can see, the general nature of the separated substances is similar, although all the eluates of the chanterelles are light yellow and the eluates No. $28-43$ 


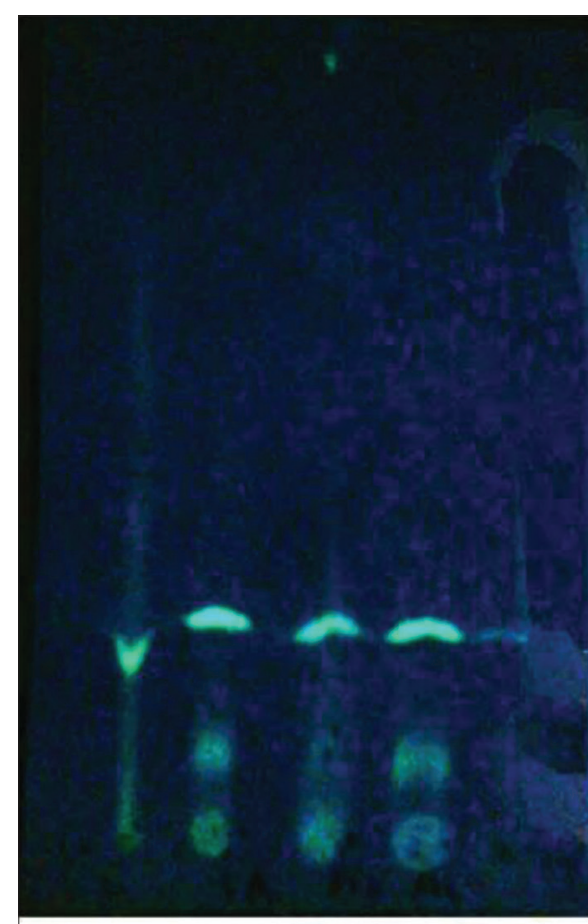

A $1 \quad 2$

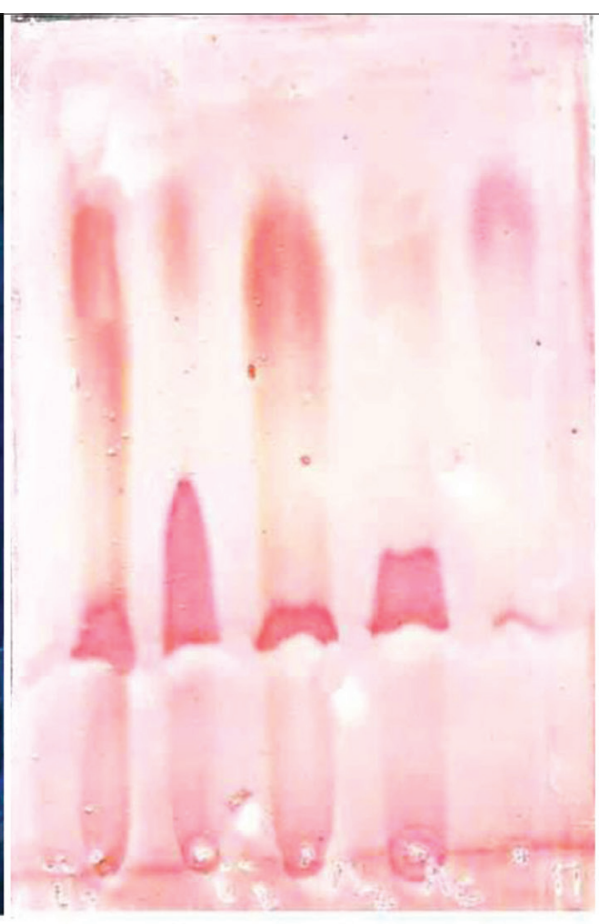

B $\begin{array}{lllll}1 & 2 & 3 & 4 & 5\end{array}$

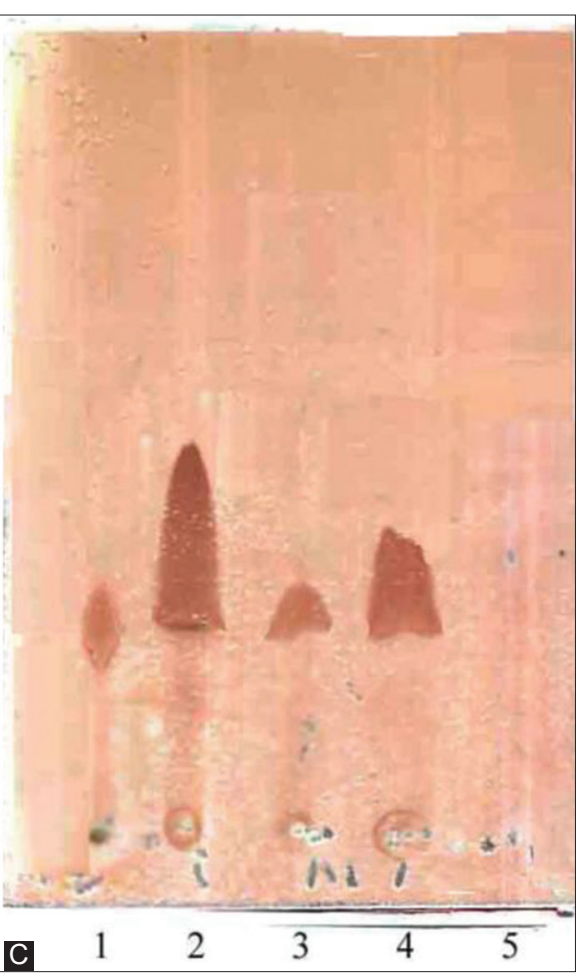

C

Fig 1. Thin layer chromatograms of hexane and alcohol extracts of Cantharellus cibarius and Hydnum repandum, A - Chromatogram in UV light; B - Chromatogram sprayed with methyl red; C - Formation of copper soaps. The chromatogram was treated with $7 \%$ copper acetate and after washing with water with $1 \%$ solution of potassium ferrocyanide. Solvent system: methylene chloride - acetic acid 100: 1. 1 - Hexane extract of Hydnum repandum; 2 - Ethanol extract of Hydnum repandum; 3 - Hexane extract of Cantharellus cibarius; 4 - Ethanol extract of Cantharellus cibarius; 5 - Palmitic acid.

\section{Table 4: Aliphatic acids and their esters in hexane extract}

\begin{tabular}{|c|c|c|c|c|c|}
\hline \multirow[t]{2}{*}{ No } & \multirow[t]{2}{*}{ Name } & \multicolumn{2}{|c|}{ Cantharellus cibarius } & \multicolumn{2}{|c|}{ Hydnum repandum } \\
\hline & & Content (\%) & Degree of precision (\%) & Content (\%) & Degree of precision (\%) \\
\hline 1 & Palmitic acid & 11.59 & 99 & 11.14 & 99 \\
\hline 2 & Linolenic acid & 31.42 & 99 & 14.83 & 62 \\
\hline 3 & Oleic acid & 17.92 & 99 & 57.89 & 99 \\
\hline 4 & Ethyl oleate & 18.78 & 99 & - & - \\
\hline 5 & Ethyl linoleate & 12.96 & 99 & - & - \\
\hline \multirow[t]{3}{*}{6} & Stearic acid & - & - & 5.64 & 98 \\
\hline & Total & 92.67 & & 89.50 & \\
\hline & Substances with a confidence level below $50 \%$ & 7.33 & $<50$ & 10.50 & $<50$ \\
\hline
\end{tabular}

from the Hedgehog mushroom are dark brown. Also, the quantitative ratio of the initial fractions, which are better soluble in chloroform, is greater in the Hedgehog mushroom, and the chanterelles have a greater proportion of the fractions leaving the column later, and, accordingly, better soluble in alcohols, in particular, in methanol.

Fat soluble vitamins, in particular tocopherols, ergocalciferols and carotenoids in lipophilic fractions of mushroom extracts may be of greatest interest.

Tocopherols are characterized by blue fluorescence in UV light (Fig. 4a) and have a characteristic UV spectrum with a maximum absorption at $285 \mathrm{~nm}$ and a minimum absorption at $254 \mathrm{~nm}$. Indeed, a comparison of the UV spectra of a sample of tocopherol acetate solution in DMSO and solutions of eluate No. 8 chanterelle and No. 9 Hedgehog mushroom gave a very similar result (Fig. 3).

In addition, when spraying chromatograms by $56 \% \mathrm{HNO}_{3}$, followed by heating to $80^{\circ} \mathrm{C}$, eluates No. 6-8 of Hydnum repandum gave a pink color, which is characteristic of tocopherols (Fig. 4c). Also, a similar result with 56\% $\mathrm{HNO}_{3}$ for eluates No. 7-9 of Cantharellus cibarius was obtained.

The quantitative content of tocopherol in hexane extracts of Hydnum repandum and Cantharellus cibarius was determined after building a calibration graph of the known 
tocopherol acetate content at $335 \mathrm{~nm}$ and determining the concentration of tocopherol in the combined eluates No. 6-14 hexane extracts of of Hydnum repandum and Cantharellus cibarius. Tocopherol was found are presented

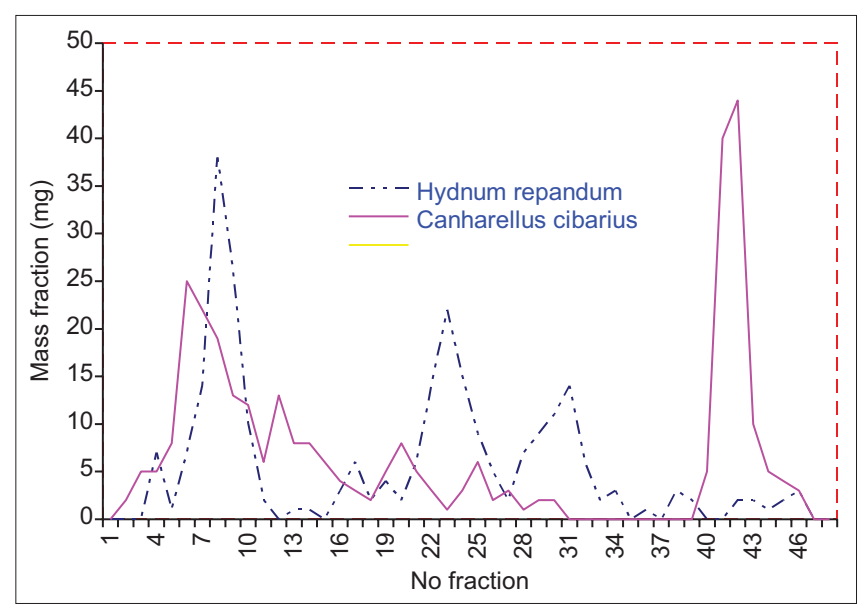

Fig 2. Separation of hexane extracts from Hydnum repandum and Cantharellus cibarius on silica gel column. $\approx 22 \%$ in the hexane eluates of Cantharellus cibarius and $17 \%$ in Hydnum repandum. This represents $\approx 0.82 \%$ of the total mass of Cantharellus cibarius ethanol extract and $\approx 0.22 \%$ of the total mass of Hydnum repandum ethanol extract.

To detect ergocalciferol on the TLC, $5 \%$ solution of phosphoric molybdic acid, $70 \%$ perchloric acid and aniline reagent were used. The most specific was the reaction with the aniline reagent (Fig. 5). These reactions indicates that ergocalciferol eluated from the $9^{\text {th }}$ to the $30^{\text {th }}$ fraction.

However, under the above conditions of chromatography, there is no complete separation of hexane extracts of hedgehog mushroom and chanterelles, in particular separation of tocopherols and ergocalciferol.

After treatment with the reagent, the chromatograms were incubated for $5 \mathrm{~min}$ at $100^{\circ} \mathrm{C}$

Manifestation of chromatograms from different fractions of hexane extracts of Hydnum repandum and Cantharellus
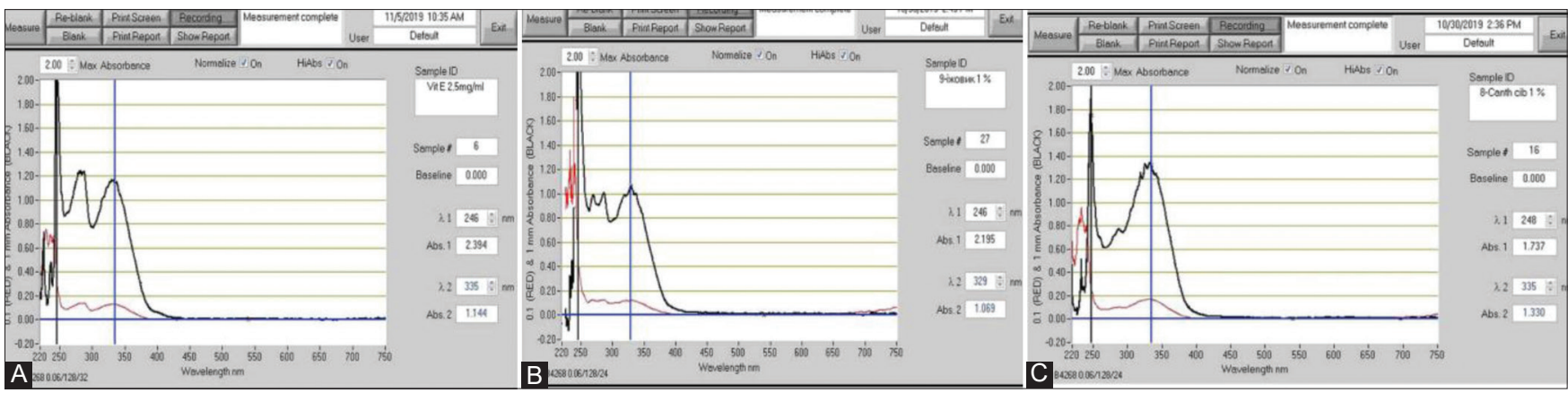

Fig 3. UV spectra of tocopherol acetate and fractions of Cantharellus cibarius and Hydnum repandum. A - Tocopherol acetate solution, $2.5 \mathrm{mg} / \mathrm{ml}$; B - Solution of fraction no 9 from Hydnum repandum, $10 \mathrm{mg} / \mathrm{ml}$; C - Solution of fraction no 8 from Cantharellus cibarius, $10 \mathrm{mg} / \mathrm{ml}$.

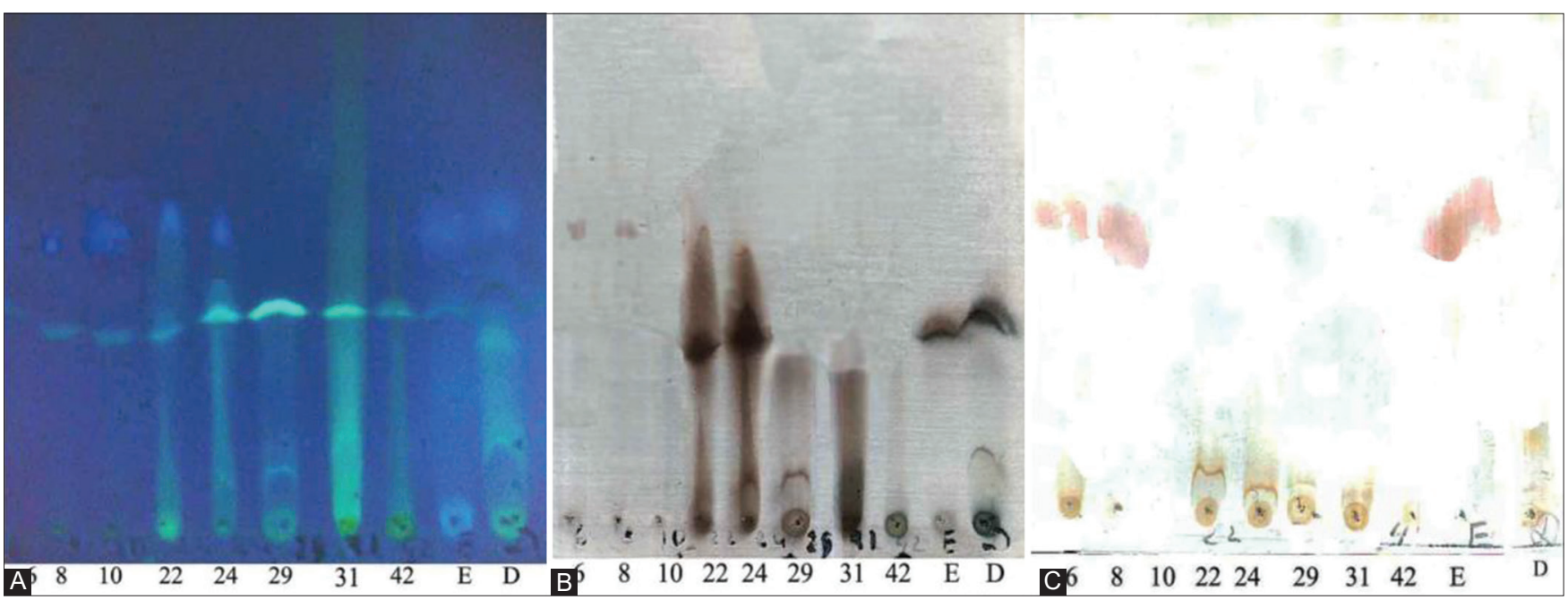

Fig 4. Hydnum repandum fractions, separated by a silica gel column, detection of tocopherols. A - Chromatogram in UV light; B - Spraying the chromatogram by $\mathrm{SbCl}_{3}$ solution; C - Spraying the chromatogram by $56 \% \mathrm{HNO}_{3}$. 6, 8, 10, 22, 24, 29, 31, 42 - fraction of Hydnum repandum obtained by chromatography of silica gel column (see Fig 2); E - tocopherol acetate, D - ergocalciferol. 
cibarius by concentrated sulfuric acid gives slightly different results (Fig. 6). In the Cantharellus cibarius present blue zones in eluates No. 12 -20, are more pronounced, whereas in the Hydnum repandum, such zones are present in fractions No. 18-21, but they are smaller and weaker. Brown and black spots show any organic matter that becomes charred in the presence of $\mathrm{H}_{2} \mathrm{SO}_{4}$.

This indicates the possible presence of carotenoids in the 12 - 20 eluates of chanterelle extracts and in the 18-21 eluates of hedgehog mushroom. Obviously, due to the presence of a large number of other substances, no characteristic absorption maxima for carotenoids in the UV spectrum were observed in the range from 400 to $550 \mathrm{~nm}$.
Reactions with the solution of stybium chloride in chloroform and spraying with TLC with phosphoric molybdic acid solution are not specific enough and can produce many other organic substances.

Modern instrumental physicochemical methods of analysis greatly simplify the task of identifying new substances. GCMS and LC-MS and HPLC are very valuable in this regard. However, in these cases, towards the obtained results the approach should be rather critical. Identification of new substances by HPLC based on retention time alone is not sufficient to formulate definitive conclusions. This is especially in the cases when nothing is known about the nature of the tested substance. When investigating the chemical composition of plant and mushroom extracts,

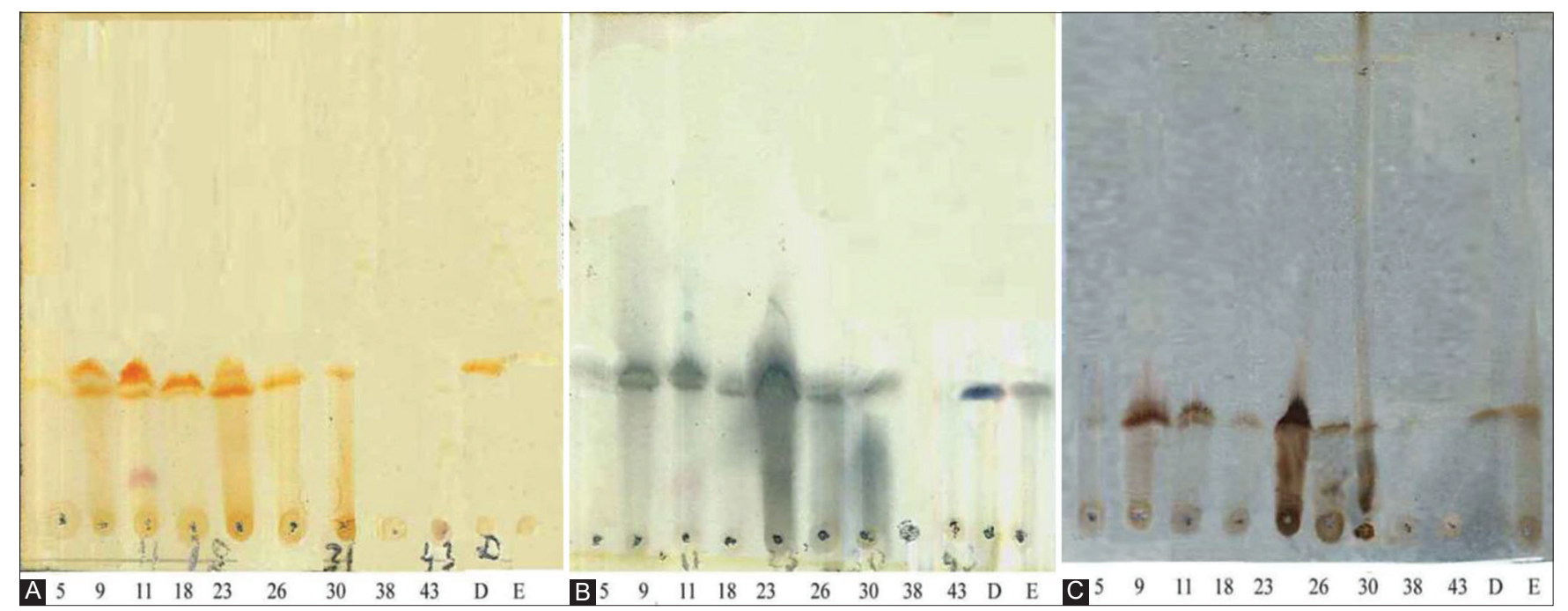

Fig 5. Detection of ergocalciferol on TLC. A - Chromatogram processing by aniline - hydrochloric acid; B - Chromatogram processing by $5 \%$ ethanolic solution of phosphonomolybdic acid; C - Chromatogram processing by $70 \% \mathrm{HClO}_{4}$; Solvent system: dichlorethane - acetic acid methanol (100:1:1); 5, 9, 11, 18, 23, 26, 30, 38, 43 - fractions of hexane extract of Hydnum repandum obtained by chromatography of silica gel column (see Fig 2).

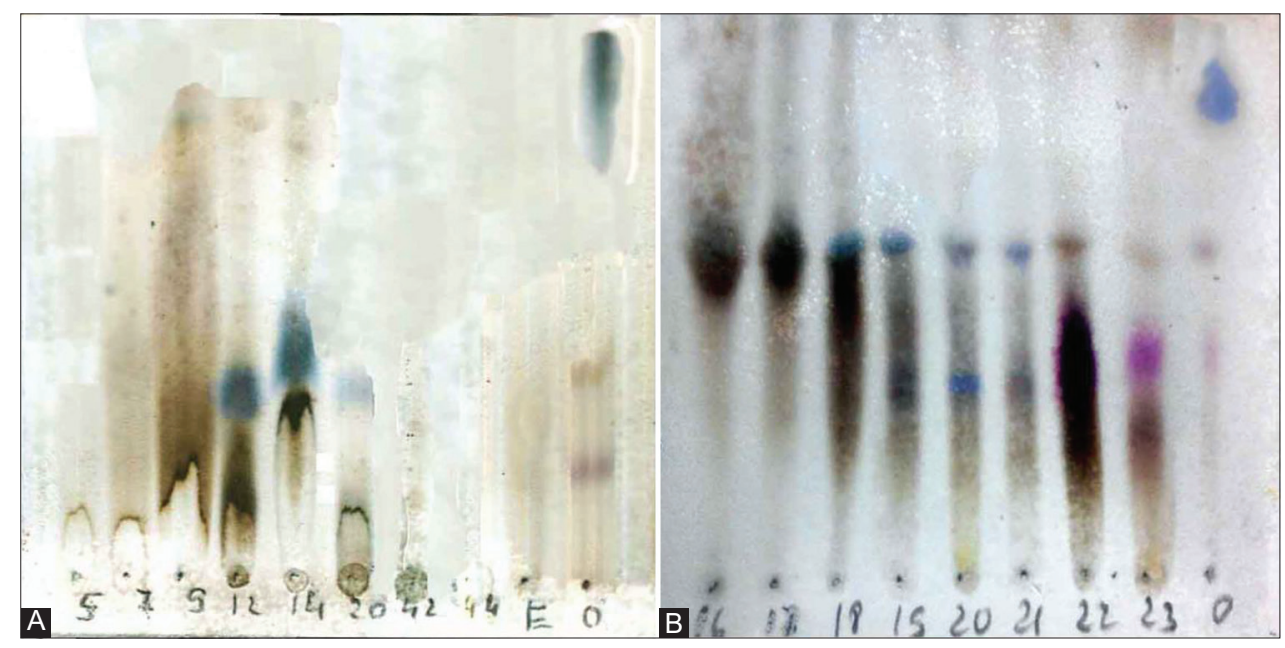

Fig 6. Treatment of TLC with sulfuric acid. A - Hexane extract from Cantharellus cibarius; B - Hexane extract from Hydnum repandum. 5, 7, 9 , 12, 14, 20, 42, 44 - fractions of hexane extracts; E - tocopherol acetate; O - oil from Sea buckthorn (Hippophae rhamnoides L.). 
there is a certain algorithm of sequential actions, which is to be followed to reduce the risk of making incorrect conclusions. It includes: 1) obtaining a number of fractions by extraction from dried raw materials with a number of polar and non-polar extractants 2) primary analysis of the obtained fractions by chemical reactions to identify groups of substances; 3) preparative chromatographic separation of the individual fractions on columns and analysis of hydrophilic fractions by paper chromatography and lipophilic fractions using TLC; 4) analysis of HPLC and GC-MS fractions.

It is clear that if the initial analysis by classical chemical reactions did not show the presence of certain groups of substances, then their further analysis by HPLC and GC-MS is pointless. Usually you can go the other way - starting with HPLC and GC-MS, by detecting of retention time to make assumptions about the presence of certain substances, then obtain them in purified form and minimally confirm the assumption at least by chemical reactions or by determining any physical- chemical characteristics. For example, Mui et al., 1998 performed detection of carotenoids of four Cantharellus species by HPLC, with the following measuring of the UV spectrum at each of the obtained peaks and comparing it to known carotenoids. Therefore, the reliability of the results obtained in this work is high.

Chanterelles are a popular food product, so there is a lot of literature regarding their chemical composition. At the same time, not all the conclusions given in these articles are sufficiently substantiated. For example, the presence of anthraquinones, flavonoids, tannins is characteristic for plants, but not of basidiomycetes. In particular, it is known that flavonoids are synthesized exclusively in higher plants (Makarenko and Levitsky, 2013; Mathesius, 2018). However, in the work of Ebrahimzadeh et al. 2015 is declared that Cantharellus cibarius hexane extract is rich in flavonoids. But glycosides and flavonoid aglycones are known to be insoluble in hexane. We have not confirmed the presence of flavonoids in the methanolic extracts of Cantharellus cibarius and Hydnum repandum. These extracts do not give the typical reactions for flavonoid compaunds and do not have UV spectrum which is characteristic for flavonoids. The conclusion that flavonoids are present in extracts based on only one reaction with $\mathrm{AlCl}_{3}$ is not sufficiently substantiated. Methanolic extracts couldn't contain proteins, as claimed by Kozarski et al., 2015, at least because proteins are insoluble in methanol. The results of the Folin-Ciocalti reaction in the analysis of phenolic compounds should be interpreted with great caution. In addition to phenolic compounds, this reaction is positive for almost all substances of protein nature and some aminoacids (tryptophan and tyrosine), as well as most primary amines (Kakac and Vejdelek, 1974), and therefore their presence must either be excluded in the analyzed extracts or their presence necessary be confirmed.

Interestingly, ethanol extracts from both mushrooms contain a high content of higher fatty acids and their esters. Re-extraction of the hexane fraction with hot $80 \%$ ethanol allowed to extract the almost pure mixture of higher fatty acids in Cantharellus cibarius and Hydnum repandum $(7.27 \%$ and $9.8 \%$, respectively, of the total mass of the extractives). Unlike higher plants, in which they are predominantly building components of fats, which are not normally extracted with $80 \%$ ethanol, these mushrooms contain fatty acids that form salts or adducts with other organic substances (eg sesquiterpenes).

Our work gives more answers to the question what substances are not present in the extracts of the fruit bodies of Cantharellus cibarius and Hydnum repandum than to the question what substances are present there. With confidence both mushrooms do not contain flavonoids, hydroxycinnamic acids, and anthraglycosides, which are characteristic of higher plants, but not for basidiomycotic mushrooms. The yellow color of Hydnum repandum and Cantharellus cibarius is not due to flavonoids. Chemical reactions confirmed the presence of carotenoids in the Cantharellus cibarius and Hydnum repandum, which are apparently responsible for their yellow color.

The ethanol extract of both mushrooms contains a high amount of low molecular weight carbohydrates and aminoacids, which make up the bulk of the extractives and determine their nutritional value.

\section{CONCLUSIONS}

We conducted research on substances that are extracted by $80 \%$ ethanol from the fruiting bodies of Cantharellus cibarius Fr. and Hydnum repandum Fr. It was found the presence of large amounts of fatty acids and their esters. Also, these mushrooms contain tocopherols, carotenoids and ergocalciferol, which are of nutritional and medicinal value. At the same time, we did not find flavonoids and hydroxycinnamic acids, which are pointed out by some authors (Barros et al., 2009; Vamanu, Nita 2014). Flavonoids and hydroxycinnamic acids are characteristic of higher plants, but are not found in basidiomycotic fungi.

\section{REFERENCES}

Akira, T., E. Takeshi and N. Shigeo. 1992. Repandiol, a new cytotoxic diepoxide from the mushrooms Hydnum repandum and H. repandum var. album. Chem. Pharm. Bull. 40: 3181-3184.

Barros, L., T. Cruz, P. Baptista, L. M. Estevinho and F. C. Ferreira. 2008. Wild and commercial mushrooms as source of nutrients 
and nutraceuticals. Food Chem. Toxicol. 46: 2742-2747.

Barros, L., M. Duenas, I. C. Ferreira, P. Baptista and C. SantosBuelga. 2009. Phenolic acids determination by HPLC-DAD-ESI/ MS in sixteen different Portuguese wild mushrooms species. Food Chem. Toxicol. 47: 1076-1079.

Botineştean, C., N. G. Hădărugă, D. I. Hădărugă and I. Jianu. 2012 Fatty acids composition by gas chromatography mass spectrometry (GC-MS) and most important physicalchemicals parameters of tomato seed oil. J. Agroaliment. Proc. Technol. 18: 89-94.

Davolia, P., A. Muccia, L. Schenettia and R. W. S. Weber. 2005. Laetiporic acids, a family of non-carotenoid polyene pigments from fruit-bodies and liquid cultures of Laetiporus sulphureus (Polyporales, Fungi). Phytochemistry. 66: 817-823.

Ebrahimzadeh, M. A., Y. Safdari and M. Khalili. 2015. Antioxidant activity of different fractions of methanolic extract of the golden chanterelle mushroom Cantharellus cibarius (Higher Basidiomycetes) from Iran. Int. J. Med. Mushrooms. 17: 557565.

Kakac, B. and Z. J. Vejdelek. 1974. Handbuch der Photometrischen Analyse organischer Verbindungen, No. Bd 1-2, Prague.

Khalili, M., M. A. Ebrahimzadeh, I. F. Omran and M. Karami. 2014. Antihypoxic activities of the golden chanterelle mushroom, Cantharellus cibarius (Higher Basidiomycetes). Int. J. Med. Mushrooms. 16: 339-344.

Kozarski, M., A. Klaus, J. Vunduk, Z. Zizak, M. Niksic, D. Jakovljevic, M. M. Vrvic and L. J. L. Van Griensven. 2015. Nutraceutical properties of the methanolic extract of edible mushroom Cantharellus cibarius (Fries): Primary mechanisms. Food Funct. 6: 1875-1886.

Long, C., X. Peng, J. Lv, S. Liao, S. Ou and Y. Shen. 2017. Purification and structural characterization of a novel watersoluble neutral polysaccharide from Cantharellus cibarius and its immunostimulating activity in RAW264.7 cells. Int. J. Polym.
Sci. 2017: 3074915.

Makarenko, O. A. and A. P. Levitsky. 2013. Physiological functions of flavonoids in plants. Physiol. Biochem. Cult. Plants. 45: 100-112.

Mathesius, U. 2018. Flavonoid functions in plants and their interactions with other organisms. Plants. 7: 30-33.

Methods of Carbohydrate Chemistry. (1967) English/ed. N.K. Kochetkova 1967. Moscow: Mir 512 (in Russian). Methods of Carbohydrate Chemistry.

Mui, D., T. Feibelmant and J. W. Bennett. 1998. A preliminary study of the carotnoids of some north American species of Cantharellus. Int. J. Plant Sci. 159: 244-248.

Nwofor, S. C., O. A. Morenikeji, A. I. O. Morenike and O. T. Oyeyemi. 2019. Inhibitory activities of ethanolic extracts of two macrofungi against eggs and miracidia of Fasciola spp. Open Life Sci. 13: 504-510.

Pochinok, H. N. 1976. Methods of Biochemical Analysis of Plants, Kiev: Naukova Dumka, p. 334.

Stryamets, N., M. Elbakidze, M. Ceuterick, P. Angelstam and R. Axelsson. 2015. From economic survival to recreation: Contemporary uses of wild food and medicine in rural Sweden, Ukraine and NW Russia. J. Ethnobiol. Ethnomed. 11: 53.

Tsyganov, M. A., G. B. Vishnivetskaya, T. P. Kukina, I. V. Sorokina, M. N. Lvova, M. A. Protsenko, N. E. Kostina and D. F. Avgustinovich. 2018. Studyng the effects of Cantharellus cibarius fungi on Opisthorchis felineus trematode and on parasite host c57BI/6 inbred mice. Vavilov J. Genet. Breed. 22: 850-858.

Valentao, P., P. B. Andrade, J. Rangel, B. Ribeiro, B. M. Silva, P. Baptista and R. M. Seabra. 2005. Effect of the conservation procedure on the contents of phenolic compounds and organic acids in chanterelle (Cantharellus cibarius) mushroom. J. Agric. Food. Chem. 53: 4925-4931.

Vamanu, E. and S. Nita. 2014. Bioactive compounds, antioxidant and anti-inflammatory activities of extracts from Cantharellus cibarius. Rev. Chim. (Bucharest). 65: 372-379. 